\title{
Cortactin contributes to the tumorigenicity of colorectal cancer by promoting cell proliferation
}

\author{
HUO WU ${ }^{*}$, XI CHENG ${ }^{*}$, XIAOPIN JI ${ }^{*}$, YONGGANG HE, XIAOQIAN JING, HAOXUAN WU and REN ZHAO \\ Department of General Surgery, Shanghai Institute of Digestive Surgery, Ruijin Hospital, \\ Shanghai Jiaotong University School of Medicine, Shanghai 200025, P.R. China
}

Received May 18, 2016; Accepted June 25, 2016

DOI: $10.3892 /$ or.2016.5207

\begin{abstract}
Cortactin is a scaffolding protein that regulates Arp2/3-mediated actin polymerization. We showed in a previous study that cortactin was highly expressed in human stage II-III colorectal cancer (CRC) tissues. In the present study, using colony formation and CCK-8 assays, we showed that overexpression of cortactin accelerated the proliferation of CRC cells. Flow cytometric assays revealed that cortactin promoted G1/S phase cell cycle transition. Later, we constructed the phosphorylation mutation of cortactin at the Tyr421 residue. Colony formation and CCK-8 assays showed that cortactin/Tyr421A lost its ability to promote cell proliferation. Western blot analysis indicated that cortactin activated cyclin D1, but not cortactin/Tyr421A. Further study in nude mice revealed that there was a greater decrease in both tumor volume and tumor weight in animals injected with SW480/cortactin/Tyr421A cells than in those injected with SW480/cortactin/WT cells. Thus, the present study demonstrates that the cortactin Tyr421 residue is required to promote cell proliferation both in vitro and in vivo.
\end{abstract}

\section{Introduction}

Colorectal cancer (CRC) is a malignancy with high incidence and mortality, placing a heavy economic burden on public health systems worldwide (1). A better understanding of the molecular mechanisms involved in CRC tumorigenesis may provide new approaches for the diagnosis and treatment of CRC.

Correspondence to: Dr Ren Zhao, Department of General Surgery, Shanghai Institute of Digestive Surgery, Ruijin Hospital, Shanghai Jiaotong University School of Medicine, 197 Rui Jin Er Road, Shanghai 200025, P.R. China

E-mail: rjzhaoren@139.com

${ }^{*}$ Contributed equally

Key words: cortactin, colorectal cancer, cyclin D1, mutation, proliferation, phosphorylation
Our laboratory previously found that cortactin is highly expressed in human stage II-III CRC and that the expression of cortactin is an independent prognostic factor for disease-free and overall survival (2). Cortactin, encoded by the CTTN (also known as EMS1) gene, is an F-actin binding protein that recruits Arp2/3 complex proteins to F-actin to regulate actin nucleation, and cytoskeletal assembly and adhesion (3). Cortactin is mainly located in the cell cortex and contains 4 major domains: the N-terminal acidic (NTA) domain, a tandem repeat region, a proline-rich region and an $\mathrm{SH} 3$ domain. The proline-rich domain includes Tyr421, Tyr466 (Tyr470 in humans) and Tyr482 (Tyr486 in humans). It is generally thought to be the signaling end of the molecule, as it contains phosphorylation sites for a number of kinases including src (4). Increased levels of cortactin are involved in the invasion and metastasis of various types of cancers, including head and neck squamous cell carcinoma (HNSCC) (5), esophageal (6), gastric (7) and hepatocellular cancer (8), melanoma (9), CRC $(2,10)$ and ovarian cancer (11). As mentioned above, cortactin plays a significant role in cancer metastasis, but little is known concerning its role in cell proliferation. Wei et al demonstrated that cortactin overexpression in gastric cancer SGC-7901 cells accelerated tumor growth by promoting EGFR expression and activating the EGFR signaling pathway (12). In the present study, we found that cortactin promoted cell proliferation by activating cyclin D1 in CRC. A mutation of cortactin at the Tyr421 phosphorylation site impaired its ability to promote CRC cell growth.

\section{Materials and methods}

Cell culture and transfection. The human CRC cell lines SW1116 and SW480 were obtained from the American Type Culture Collection (ATCC; Manassas, VA, USA). These cell lines were cultured in RPMI-1640 medium. All media were supplemented with $10 \%$ fetal calf serum, $100 \mathrm{U} / \mathrm{ml}$ penicillin, and $100 \mu \mathrm{g} / \mathrm{ml}$ streptomycin. They were then cultured at $37^{\circ} \mathrm{C}$ with $5 \% \mathrm{CO}_{2}$. The plasmids for cortactin knockdown and lentivirus particles for cortactin overexpression were constructed by Shanghai GenePharma (Shanghai, China). A mutated form of cortactin (mutation at the Tyr421 phosphorylation site, Tyr mutant to alanine with flag as tag) was obtained from Shanghai USEN Biological Technology (Shanghai, China). The plasmids and lentivirus were transfected according to the 
manufacturer's guidelines. Puromycin was used for stable cell screening and establishment. The transfection efficiency was detected by western blotting.

Westen blotting. Total cell proteins were ground and lysed in RIPA lysis buffer with $1 \%$ phenylmethylsulfonyl fluoride (PMSF). Total protein concentration was measured using a BCA assay kit (Pierce, Rockford, IL, USA). Equal amounts of protein $(50 \mu \mathrm{g})$ were subjected to $10 \%$ SDS-PAGE and transferred electrophoretically to polyvinylidene difluoride (PVDF) membranes. The membranes were blocked and then incubated with the primary antibodies overnight at $4^{\circ} \mathrm{C}$. Primary antibodies used were as follows: anti-cortactin (dilution 1:1,000; Santa Cruz Biotechnology, Santa Cruz, CA, USA), anti-cyclin D1 (dilution 1:1,000), antiflag (dilution 1:1,000) (both from Cell Signaling Technology, Danvers, MA, USA), and anti-GAPDH (dilution 1:10,000) (Abcam, Cambridge, MA, USA). After the proteins were incubated in secondary antibodies for $2 \mathrm{~h}$, signals were detected by an enhanced chemiluminescence detection system (Amersham Biosciences, Piscataway, NJ, USA).

Colony formation assay. Equal numbers of cells $\left(1.5 \times 10^{3} /\right.$ well $)$ were seeded into 6 -well plates and cultured for 2 weeks in RPMI-1640 medium. After being washed with $1 \%$ phosphate-buffered saline (PBS), the cells were fixed with methanol and stained with $0.4 \%$ crystal violet. After being carefully washed a second time with $1 \%$ PBS, images were captured and the number of clones was counted.

Cell Counting Kit-8 (CCK-8) assay. Equal number of cells (100 $\mu \mathrm{l}, 4 \times 10^{3} /$ well) were seeded into 96 -well plates in replicates of 6 . At the appropriate time $(0,24,48,72$ and $96 \mathrm{~h})$, $10 \mu \mathrm{l}$ of CCK-8 (Dojindo, Tokyo, Japan) was added to the cells. After being incubated for $2 \mathrm{~h}$ at $37^{\circ} \mathrm{C}$, the cell viability was measured at a wavelength of $450 \mathrm{~nm}$.

Cell cycle analysis. For cell cycle analysis, cells were fixed with $70 \%$ ethanol and stored overnight at $4^{\circ} \mathrm{C}$. After being washed with PBS twice, they were stained with propidium iodide (PI) solution. Cells were filtered and analyzed with flow cytometry (Beckman Instruments, Brea, CA, USA) according to the manufacturer's instructions.

Xenograft model. BALB/c nude mice at the age of 4 weeks were purchased from the Institute of Zoology of the Chinese Academy of Sciences, and housed in a dedicated SPF facility at the Laboratory Animal Center of Ruijin Hospital. SW480/cortactin/WT cells $\left(2 \times 10^{6}\right)$ were resuspended in $100 \mu 1$ PBS and injected into the left side of each nude mouse. Equal numbers of SW480/cortactin/Tyr421A cells were injected into the right sides of other nude mice. Tumor length (L) and width (W) were measured every week, and tumor volume (V) was evaluated using the following formula: $\mathrm{V}=\mathrm{LW}^{2} \pi / 6$. Tumors were weighed and photographed 28 days after injection. Serial $4.0-\mu \mathrm{m}$ sections were cut and stained with hematoxylin and eosin. The sections were further analyzed using immunohistochemistry staining. All experiments were performed in accordance with the official recommendations of the Chinese Animal Research Committee.
Immunohistochemistry. Tissues were sectioned and deparaffinized in xylene. After being rehydrated in graded ethanol, the sections were incubated in hydrogen peroxide and blocked in $10 \%$ goat serum. The sections were incubated with Ki67 antibody (dilution 1:150; Abcam) overnight at $4^{\circ} \mathrm{C}$, and incubated with the secondary antibody for $1 \mathrm{~h}$ at room temperature. Finally, the sections were incubated with diaminobenzidine and counterstained with hematoxylin.

Statistical analyses. All data were analyzed by SPSS 17.0 and are presented as mean \pm SD. Statistical differences between two groups were examined by the Student's t-test. $P<0.05$ was considered to indicate a statistically significant result. $\mathrm{P}<0.01$ was considered highly significant.

\section{Results}

Cortactin promotes the proliferation of CRC cells. To evaluate the role of cortactin in CRC cells, cortactin expression was stably increased in the SW480 cells by lentivirus infection and reduced in the SW1116 cells by plasmid infection (Fig. 1A). The CCK-8 assay showed that overexpression of cortactin significantly increased the proliferation rate of the SW480 cells, while depletion of cortactin reduced the proliferation rate of the SW1116 cells (Fig. 1B). Similar results were observed in the colony formation assays (Fig. 1C and D). Our results indicate that cortactin plays an important role in promoting $\mathrm{CRC}$ proliferation and growth.

Cortactin promotes the Gl/S phase transition of CRC cells. To explore the mechanism underlying the promotion of cellular proliferation by cortactin, a flow cytometric analysis was performed. The number of cells in the G0/G1, S and G2/M phase, respectively, was analyzed. As shown in Fig. 2A, overexpression of cortactin induced the increased percentage of G1 phase cells and significantly decreased the percentage of S phase cells in the SW480 cell line. We obtained the opposite results after cortactin knockdown in the SW1116 cells (Fig. 2B). These results indicate that cortactin promotes the progression of CRC cells in the G1/S phase.

Tyr421 mutation impairs the growth-promoting activity of cortactin in CRC cells. The proline-rich region of cortactin is a major regulatory phosphorylation motif that is targeted by a large number of protein kinases including Src and Erk $(13,14)$. Tyrosine phosphorylation of cortactin occurs at 3 tyrosine residues, Tyr421, Tyr466 (Tyr470 in humans) and Tyr482 (Tyr486 in humans), with Tyr466 and Tyr482 phosphorylation dependent on the phosphorylation of Tyr421 (15). To evaluate the impact of the phosphorylation site of cortactin on CRC cell proliferation, a mutation of cortactin at the Tyr421 site was constructed (Fig. 3A). As shown in Fig. 3B, compared with SW480/cortactin/WT, SW480/cortactin/Tyr421A exhibited a significantly lower growth rate from day 3 of cell culture. Furthermore, in the colony formation assay, the mutation of cortactin in SW480 cells resulted in reduced colony numbers as compared with the SW480/cortactin/WT cells (Fig. 3C and D). These data suggest that the Tyr421 site of cortactin is required to promote cell proliferation and growth. 
A

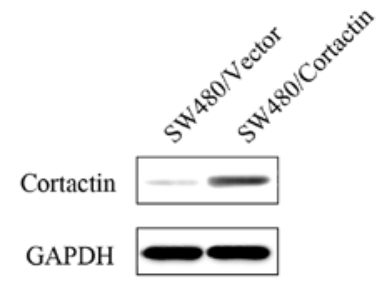

B

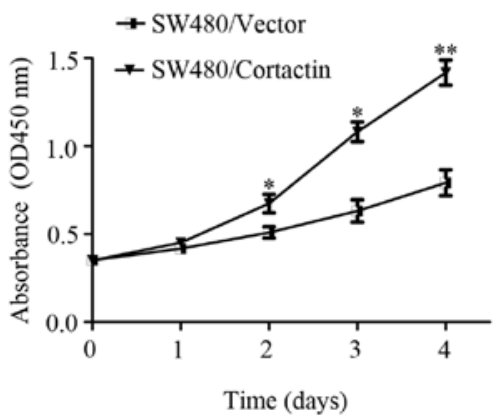

$\mathrm{C}$

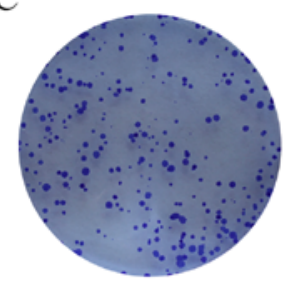

SW480/Vector

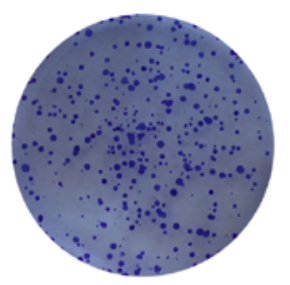

SW480/Cortactin

$\mathrm{D}$

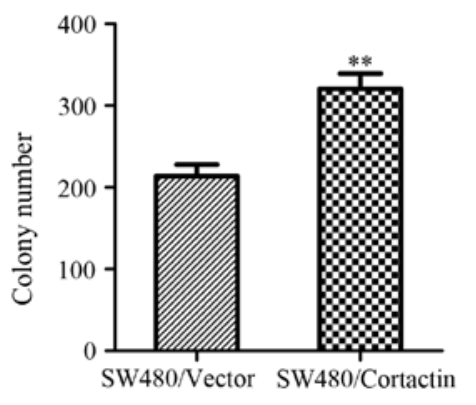

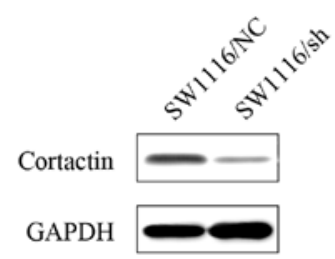
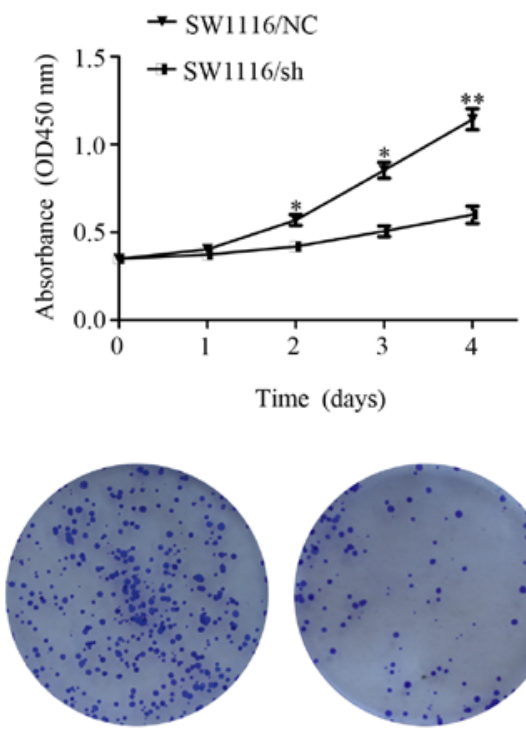

SW1116/NC

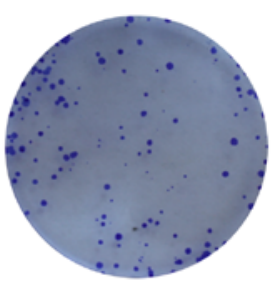

SW1116/sh

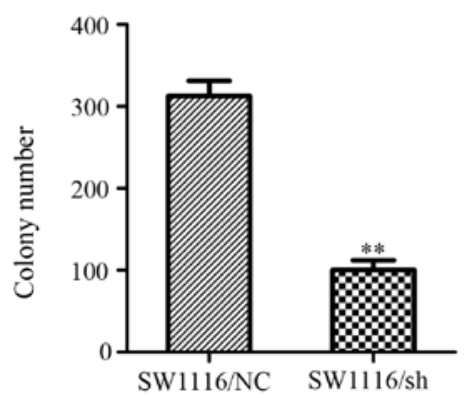

Figure 1. Cortactin promotes cell proliferation and colony formation. (A) Western blot analysis showed that cortactin was stably increased in the SW480 cells and knocked down in the SW1116 cells. (B) CCK-8 assay revealed that cortactin overexpression promoted the proliferation of SW480 cells while cortactin knockdown inhibited the proliferation of SW1116 cells. (C and D) A colony formation assay revealed that cortactin overexpression promoted colony formation of SW480 cells while cortactin knockdown inhibited colony formation of SW1116 cells. Data are the mean \pm SD of 3 independent experiments; "P<0.05 and ${ }^{* * *} \mathrm{P}<0.01$.

The Tyr421 mutation affects the ability of cortactin to promote cell cycle progression. To further validate the effects of cortactin on CRC cell proliferation, we explored the function of Tyr421 in cell cycle progression. Flow cytometric analysis showed that there was an increase in the percentage of cells in the G1 phase in the SW480/cortactin/ Tyr421A cells as compared with this percentage in the SW480/ cortactin/WT cells, while the percentage of cells at the S peak decreased when there was a mutation of cortactin at the Tyr421 site (Fig. 4A and B). These results indicate that the mutation of cortactin at the Tyr421 site caused G1 arrest in the CRC cell cycle.

Cortactin induces cyclin D1 expression. Having found that cortactin promoted the progression of the G1/S phase, we sought to determine whether this was due to changes in cell cycle-related protein. Therefore, western blot analysis was performed to assess the correlation between the effects of cortactin on the expression of cyclin D1. As shown in Fig. 5A (in line with the results of our clone formation assay, CCK-8 assay, and cell cycle analysis) ectopic cortactin in SW480 cells increased the expression level of the cell cycle-related protein cyclin D1, while silencing of cortactin in the SW1116 cells produced the opposite result. Notably, cyclin D1 was further enhanced by the overexpression of cortactin/WT in the SW480 cells but not by the mutation of cortactin at the Tyr421 site (Fig. 5B). These data indicate that the Tyr421 site of cortactin is required to induce cyclin D1.

The Tyr421 mutation impairs tumorigenesis in vivo. To further determine the role of Tyr421 in the tumorigenicity of CRC, equal numbers of SW480/cortactin/WT and SW480/cortactin/ 


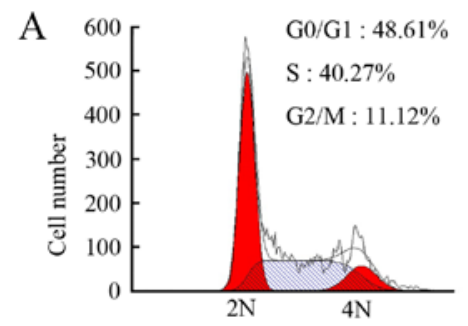

SW480/Vector

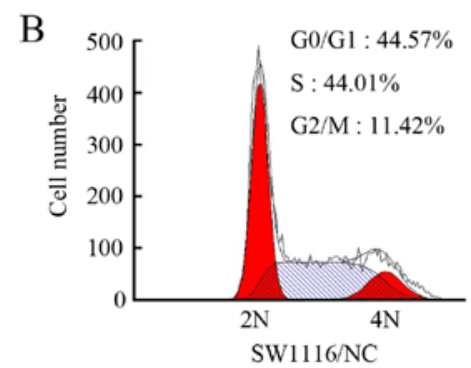

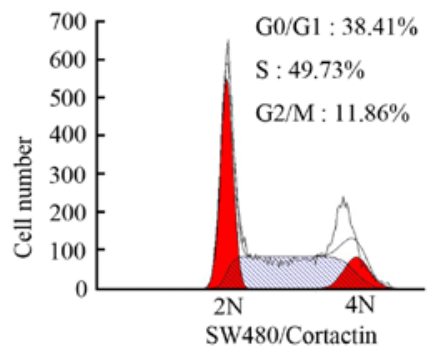

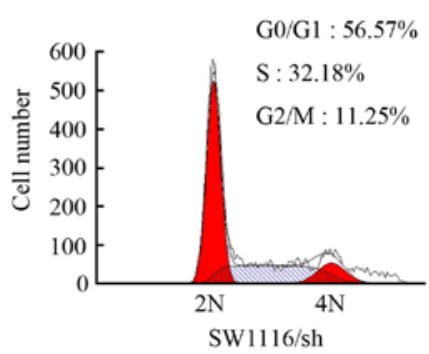

$\mathrm{C}$

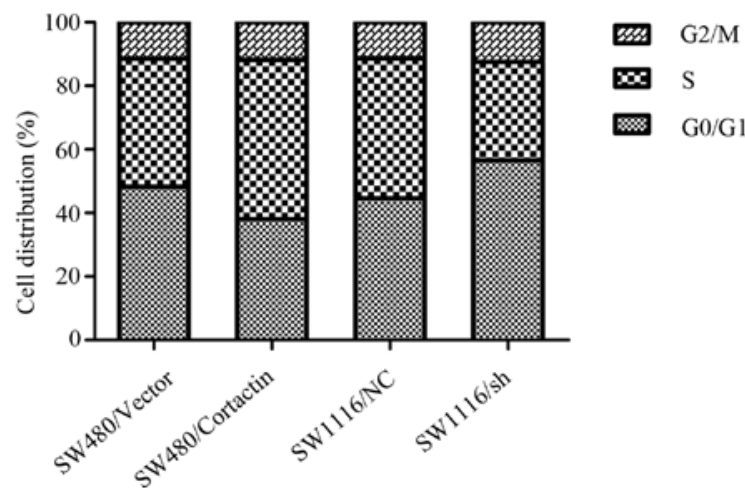

Figure 2. Cortactin promotes the G1/S transition of CRC cells. (A) Flow cytometric analysis found that cortactin overexpression in SW480 cells induced the reduction of cells in the G1 phase. (B) Flow cytometric analysis found that cortactin knockdown in SW1116 cells induced an increase in cells in the G1 phase. (C) The proportion of cells in various phases of the indicated cell cycle. Data are the mean \pm SD of 3 independent experiments.

A

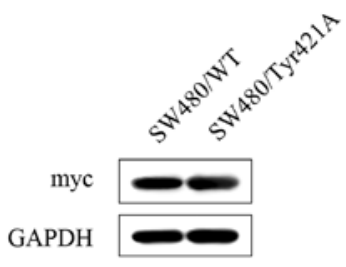

$\mathrm{C}$

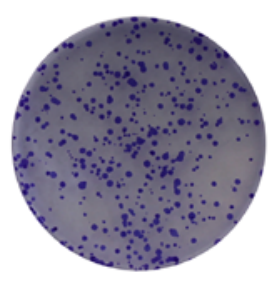

SW480/WT
B

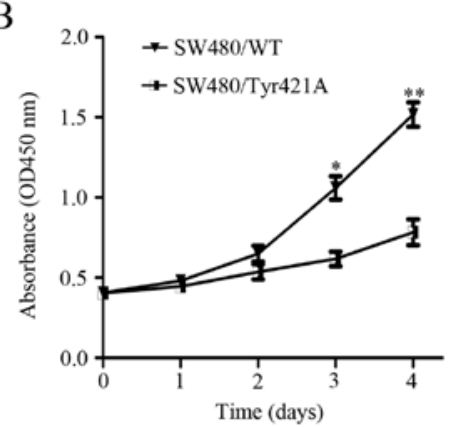

$\mathrm{D}$

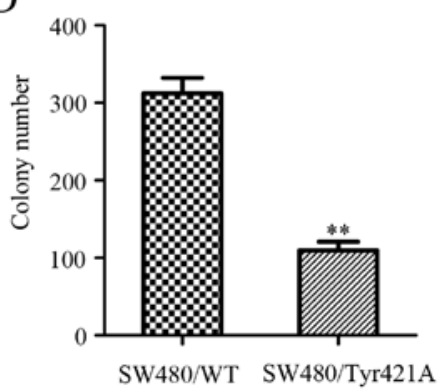

Figure 3. The Tyr421A mutation affects the ability of cortactin to promote the proliferation of CRC cells. (A) The expression of myc-tagged fragments of cortactin as demonstrated by western blot analysis. (B) The proliferation of SW480/cortactin/WT and SW480/cortactin/Tyr421A cells as determined by CCK-8 assay. (C and D) The proliferation of SW480/cortactin/WT and SW480/cortactin/Tyr421A cells as determined by colony formation assay. Data are mean \pm SD of 3 independent experiments; ${ }^{*} \mathrm{P}<0.05,{ }^{* *} \mathrm{P}<0.01$. 

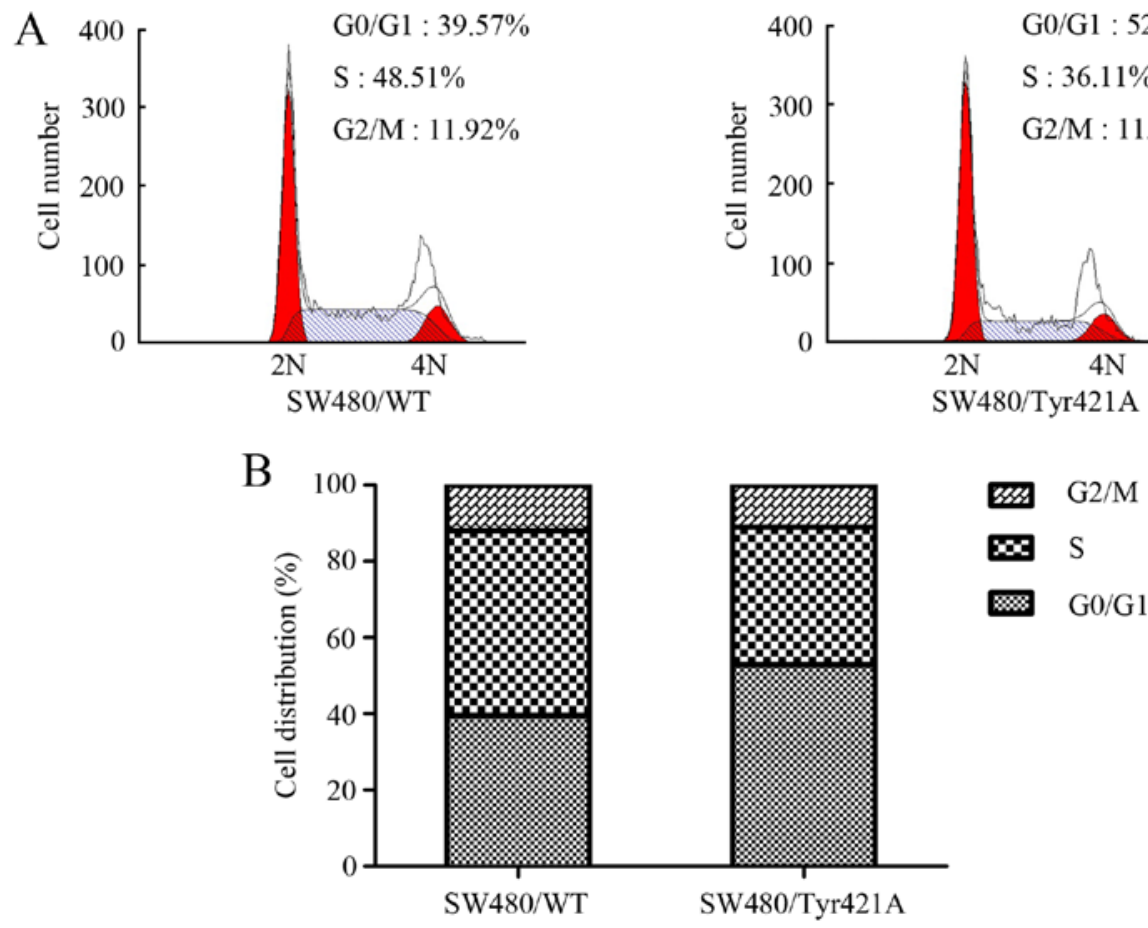

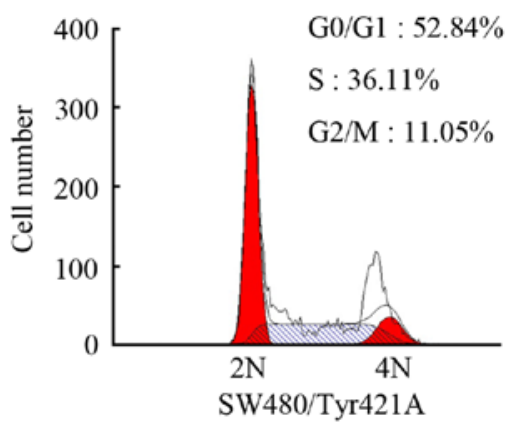

$\mathrm{G} 2 / \mathrm{M}$

$\mathrm{G} 0 / \mathrm{G} 1$

Figure 4. The Tyr421A mutation affects the ability of cortactin to promote cell cycle progression of CRC cells. (A) Flow cytometric analysis found that the Tyr421A mutation in SW480 cells increased the percentage of cells in the G1 phase. (B) The proportion of cells in the various phases of the SW480/cortactin/ WT and SW480/cortactin/Tyr421A cell cycle. Data are mean \pm SD of 3 independent experiments.

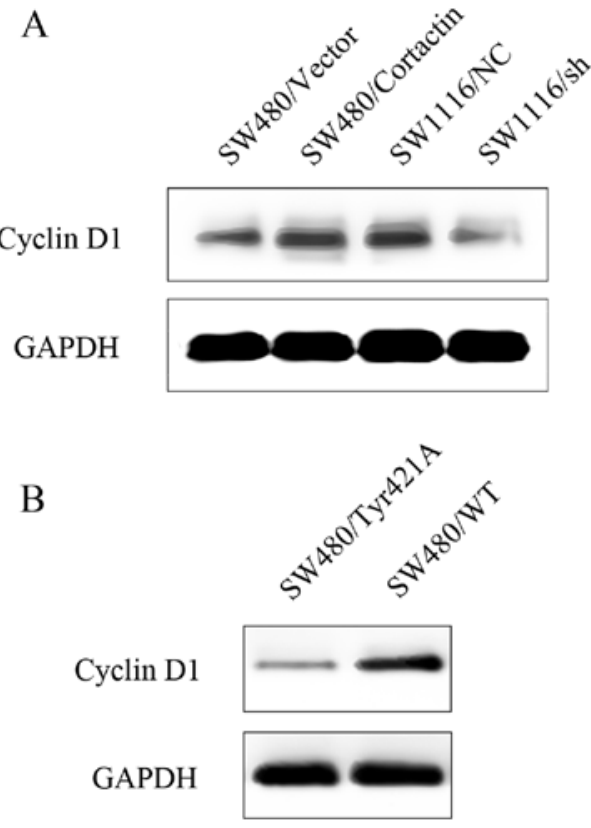

Figure 5. Cortactin regulates the expression of cyclin D1. (A) Western blot analysis determined that cortactin promoted the expression of cyclin D1. (B) The expression of cyclin D1 in the SW480/cortactin/WT and SW480/cortactin/Tyr421A cells. Data are the mean \pm SD of 3 independent experiments.

Tyr421A cells were injected subcutaneously into the flanks of 4-week BALB/c nude mice. Tumor volumes were analyzed every week, and tumor grafts were excised and weighed after 28 days. As shown in Fig. 6A and B, tumor growth of the SW480/cortactin/Tyr421A cells was slower than that of the SW480/cortactin/WT cells. Moreover, the average tumor weight in the SW480/cortactin/Tyr421A cell group was significantly lower than that in the SW480/cortactin/WT cell group (Fig. 6B). Immunohistochemical analysis revealed that SW480/cortactin/WT tumors displayed a higher Ki67 index, whereas SW480/cortactin/Tyr421A tumors showed reduced numbers of Ki67-positive cells (Fig. 6D). Taken together, these results suggest that cortactin promotes the tumorigenicity of CRC cells in vivo.

\section{Discussion}

Colorectal cancer (CRC) is a malignant tumor of high morbidity and mortality (1). Cell proliferation is an obvious characteristic of malignant tumors. The growth of cells involves a series of mechanisms wherein cell cycle arrest plays an important role, being responsible for the survival of the cell growth process (16). Targeting the deregulated cell cycle is a practical strategy by which to check uncontrolled proliferation in cancer cells. In the present study, we demonstrated that cortactin regulated the G1/S checkpoint and promoted the proliferation of CRC cells both in vitro and in vivo.

Cortactin was first identified as a tyrosine phosphorylated protein in Src-transformed primary chicken embryo cells (17). The gene for cortactin is in the chromosome 11q13 region, which is frequently amplified in human cancers (18). Cortactin is a scaffolding protein that activates the Arp2/3 complex through its NTA region, which promotes the branching of actin filaments and stabilizes branched networks. The cortactin SH3 domain interacts with a number of proteins that regulate membrane trafficking and cell motility (19). Cortactin is overexpressed in a large number of cancers and appears to 
A

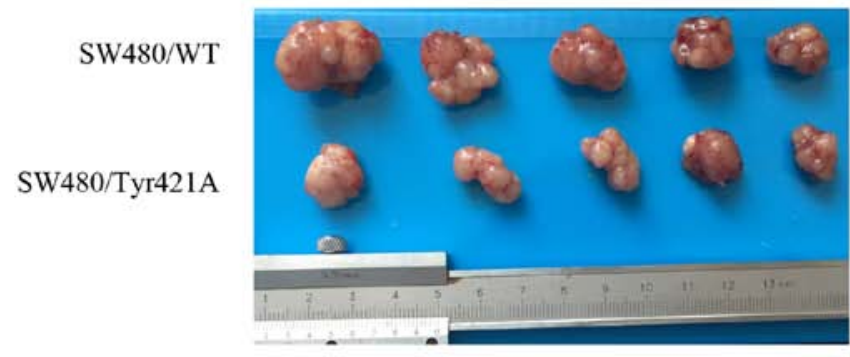

B

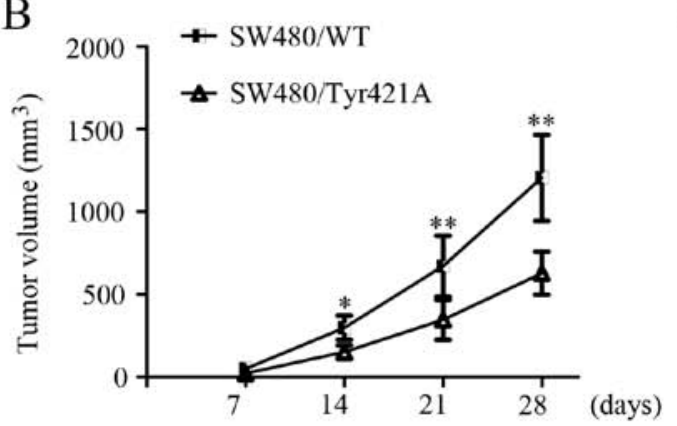

$\mathrm{C}$

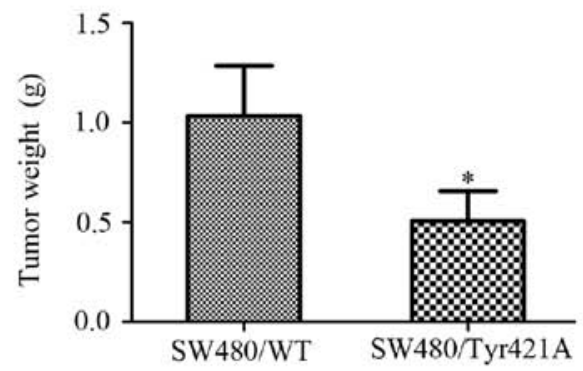

$\mathrm{D}$

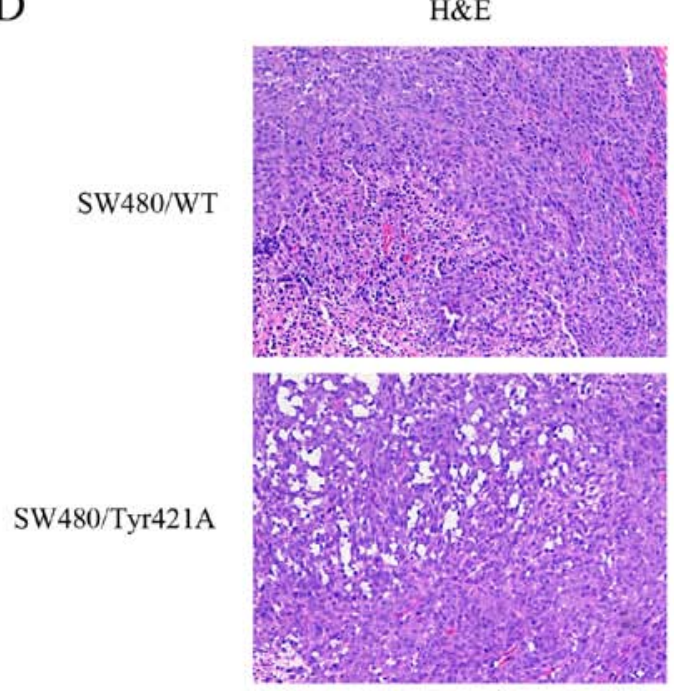

Ki67
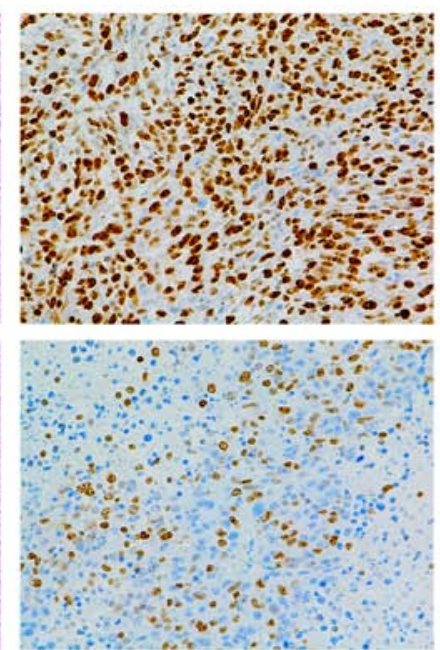

Figure 6. Effects of cortactin/Tyr421 on tumor growth in vivo. (A) Representative images of tumors in nude mice (N=5/group) derived from SW480/cortactin/WT and SW480/cortactin/Tyr421A cells. (B) Tumor volume was monitored weekly after treatment with SW480/cortactin/WT and SW480/cortactin/Tyr421A cells in nude mice. (C) Average tumor weights derived from SW480/cortactin/WT and SW480/cortactin/Tyr421A cells. (D) Representative images of H\&E staining and Ki67 expression of tumor xenograft. Data are the mean $\pm \mathrm{SD}$ of 3 independent experiments; ${ }^{*} \mathrm{P}<0.05,{ }^{* *} \mathrm{P}<0.01$.

be an attractive target owing to its prominent role in the formation and function of invadopodia as well as in the promotion of the migration and invasion of cancer cells (20). Despite the numerous studies that have been carried out, the role of cortactin in cancer growth still requires further research. Li et al indicated that compared with CortF421F466F482, cortactin contributes to the metastasis of breast cancer, but has no significant effects on the growth of cancer cells (21). Ni et al found that cortactin is significantly upregulated in colon cancer tissues and that the expression of ectopic cortactin in HCT116 cells promoted cell proliferation and tumorigenicity (22). The results presented in the present study revealed that ectopic expression of cortactin in the SW480 cells promoted cell proliferation and colony formation, while knockdown of cortactin in SW1116 cells produced the opposite results.
Tyrosine phosphorylation of cortactin occurs on Tyr421, Tyr466 (Tyr470 in humans) and Tyr482 (Tyr486 in humans) residues located within the proline-rich domain in response to Src and several other tyrosine kinases $(4,13,23)$. Notably, tyrosine phosphorylation of cortactin is a progressive process among the 2 main residues, with pTyr421 required for the phosphorylation of Tyr466 (15). Recently Radhakrishnan et al demonstrated that pTyr421 cortactin is highly expressed in colon cancer, and that curcumin significantly reduced the level of pTyr421 cortactin by a direct physical interaction with PTPN1. This ultimately led to a decrease in the migration of colon cancer cells (24). To the best of our knowledge, the role of Tyr421 cortactin in cell proliferation has not yet been studied. The results presented in the present study showed that the Tyr421 mutation of cortactin impaired its growth-supporting 
activity both in vitro and in vivo. It may be of great interest to investigate whether the Tyr470 mutation of cortactin could regulate the proliferation of cancer cells. To better understand how cortactin promotes the growth of CRC cells, we analyzed the impact of cortactin on cell cycle progression. We found that the silencing of cortactin with shRNA resulted in an accumulation of cells in the G0/G1 phase, leading to G1/S cell cycle arrest in the SW116 cells. In contrast, overexpression of cortactin facilitated the G1/S transition in the SW480 cells. Notably, the mutation of cortactin at the Tyr421 site in the SW480 cells caused a G1 arrest in the cell cycle. Cylin D1 has been reported to promote cell progression by inducing cell transition from G0/G1 to S phase (25). Recent research has shown that cortactin enhances EGFR expression and stimulates the EGFR-ERK signaling pathway (22). A previous study reported that the ERK/cyclin D1 pathway is involved in the proliferation of smooth muscle cells (26). With this inference, our results indicate that cortactin promotes the G1/S transition by regulating cylin D1. Mutation of cortactin at the Tyr421 residue impaired the activation of cyclin D1, indicating that the cortactin regulation of cyclin D1 is dependent on Tyr421 phosphorylation. However, further studies are required to identify the detailed interaction between cortactin and cyclin D1. In conclusion, the present study demonstrated that cortactin plays an important role in CRC proliferation and tumorigenicity, suggesting the possibility of a new therapeutic option.

\section{Acknowledgements}

The present study was supported by the National Natural Science Foundation of China (no. 81272751).

\section{References}

1. Siegel RL, Miller KD and Jemal A: Cancer statistics, 2016. CA Cancer J Clin 66: 7-30, 2016.

2. Cai JH, Zhao R, Zhu JW, Jin XL, Wan FJ, Liu K, Ji XP, Zhu YB and Zhu ZG: Expression of cortactin correlates with a poor prognosis in patients with stages II-III colorectal adenocarcinoma. J Gastrointest Surg 14: 1248-1257, 2010.

3. van Rossum AG, Moolenaar WH and Schuuring E: Cortactin affects cell migration by regulating intercellular adhesion and cell spreading. Exp Cell Res 312: 1658-1670, 2006.

4. Weaver AM: Cortactin in tumor invasiveness. Cancer Lett 265: $157-166,2008$

5. Rodrigo JP, García LA, Ramos S, Lazo PS and Suárez C: EMSI gene amplification correlates with poor prognosis in squamous cell carcinomas of the head and neck. Clin Cancer Res 6: 3177-3182, 2000

6. Lu P, Qiao J, He W, Wang J, Jia Y, Sun Y, Tang S, Fu L and Qin Y: Genome-wide gene expression profile analyses identify CTTN as a potential prognostic marker in esophageal cancer. PLoS One 9: e88918, 2014.

7. Wang X, Cao W, Mo M, Wang W, Wu H and Wang J: VEGF and cortactin expression are independent predictors of tumor recurrence following curative resection of gastric cancer. J Surg Oncol 102: 325-330, 2010.
8. Yuan BZ, Zhou X, Zimonjic DB, Durkin ME and Popescu NC: Amplification and overexpression of the EMS 1 oncogene, a possible prognostic marker, in human hepatocellular carcinoma. J Mol Diagn 5: 48-53, 2003.

9. Xu XZ, Garcia MV, Li TY, Khor LY, Gajapathy RS, Spittle C, Weed S, Lessin SR and Wu H: Cytoskeleton alterations in melanoma: Aberrant expression of cortactin, an actin-binding adapter protein, correlates with melanocytic tumor progression. Mod Pathol 23: 187-196, 2010.

10. Hirakawa H, Shibata $K$ and Nakayama T: Localization of cortactin is associated with colorectal cancer development. Int $\mathbf{J}$ Oncol 35: 1271-1276, 2009.

11. Li A, Zhang L, Zhang X, Jin W and Ren Y: Expression and clinical significance of cortactin protein in ovarian neoplasms. Clin Transl Oncol 18: 220-227, 2016.

12. Wei J, Zhao ZX, Li Y, Zhou ZQ and You TG: Cortactin expression confers a more malignant phenotype to gastric cancer SGC-7901 cells. World J Gastroenterol 20: 3287-3300, 2014.

13. Mezi S, Todi L, Orsi E, Angeloni A and Mancini P: Involvement of the Src-cortactin pathway in migration induced by IGF-1 and EGF in human breast cancer cells. Int J Oncol 41: 2128-2138, 2012.

14. Martinez-Quiles N, Ho HY, Kirschner MW, Ramesh N and Geha RS: Erk/Src phosphorylation of cortactin acts as a switch on-switch off mechanism that controls its ability to activate N-WASP. Mol Cell Biol 24: 5269-5280, 2004.

15. Head JA, Jiang D, Li M, Zorn LJ, Schaefer EM, Parsons JT and Weed SA: Cortactin tyrosine phosphorylation requires Rac1 activity and association with the cortical actin cytoskeleton. Mol Biol Cell 14: 3216-3229, 2003.

16. Hanahan D and Weinberg RA: Hallmarks of cancer: The next generation. Cell 144: 646-674, 2011.

17. Kanner SB, Reynolds AB, Vines RR and Parsons JT: Monoclonal antibodies to individual tyrosine-phosphorylated protein substrates of oncogene-encoded tyrosine kinases. Proc Natl Acad Sci USA 87: 3328-3332, 1990.

18. Schuuring E: The involvement of the chromosome $11 \mathrm{q} 13$ region in human malignancies: Cyclin DI and EMSI are two new candidate oncogenes - a review. Gene 159: 83-96, 1995.

19. Weed SA and Parsons JT: Cortactin: Coupling membrane dynamics to cortical actin assembly. Oncogene 20: 6418-6434, 2001.

20. MacGrath SM and Koleske AJ: Cortactin in cell migration and cancer at a glance. J Cell Sci 125: 1621-1626, 2012.

21. Li Y, Tondravi M, Liu J, Smith E, Haudenschild CC, Kaczmarek M and Zhan X: Cortactin potentiates bone metastasis of breast cancer cells. Cancer Res 61: 6906-6911, 2001.

22. Ni QF, Yu JW, Qian F, Sun NZ, Xiao JJ and Zhu JW: Cortactin promotes colon cancer progression by regulating ERK pathway. Int J Oncol 47: 1034-1042, 2015.

23. Boyle SN, Michaud GA, Schweitzer B, Predki PF and Koleske AJ: A critical role for cortactin phosphorylation by Abl-family kinases in PDGF-induced dorsal-wave formation. Curr Biol 17: 445-451, 2007.

24. Radhakrishnan VM, Kojs P, Young G, Ramalingam R, Jagadish B, Mash EA, Martinez JD, Ghishan FK and Kiela PR: pTyr $^{421}$ cortactin is overexpressed in colon cancer and is dephosphorylated by curcumin: Involvement of non-receptor type 1 protein tyrosine phosphatase (PTPN1). PLoS One 9: e85796, 2014.

25. Resnitzky D and Reed SI: Different roles for cyclins D1 and E in regulation of the $\mathrm{G}_{1}$-to-S transition. Mol Cell Biol 15: 3463-3469, 1995.

26. Li T, Song T, Ni L, Yang G, Song X, Wu L, Liu B and Liu C: The p-ERK-p-c-Jun-cyclinD1 pathway is involved in proliferation of smooth muscle cells after exposure to cigarette smoke extract. Biochem Biophys Res Commun 453: 316-320, 2014. 